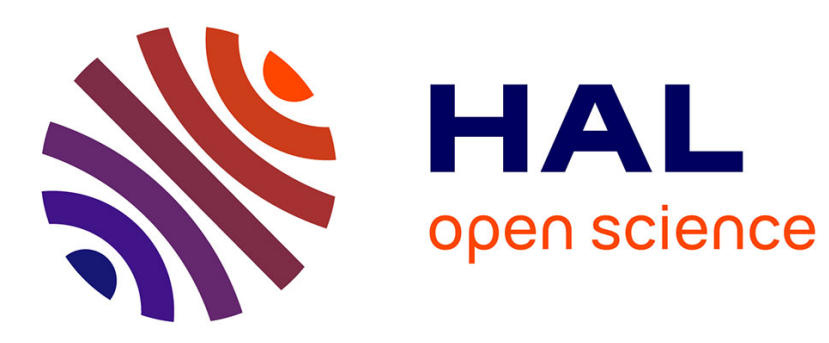

\title{
Examining the impact of multi-layer graphene using cellular and amphibian models
}

\author{
Laura Muzi, Florence Mouchet, Stéphanie Cadarsi, Izabela Janowska, Julie \\ Russier, Cécilia Ménard-Moyon, Gianfranco Risuleo, Brigitte Soula, \\ Anne-Marie Galibert, Emmanuel Flahaut, et al.
}

\section{To cite this version:}

Laura Muzi, Florence Mouchet, Stéphanie Cadarsi, Izabela Janowska, Julie Russier, et al.. Examining the impact of multi-layer graphene using cellular and amphibian models. 2D Materials, 2016, vol. 3 $\left(\mathrm{n}^{\circ} 2\right)$, pp. 025009. 10.1088/2053-1583/3/2/025009 . hal-01462915

\section{HAL Id: hal-01462915 https://hal.science/hal-01462915}

Submitted on 9 Feb 2017

HAL is a multi-disciplinary open access archive for the deposit and dissemination of scientific research documents, whether they are published or not. The documents may come from teaching and research institutions in France or abroad, or from public or private research centers.
L'archive ouverte pluridisciplinaire HAL, est destinée au dépôt et à la diffusion de documents scientifiques de niveau recherche, publiés ou non, émanant des établissements d'enseignement et de recherche français ou étrangers, des laboratoires publics ou privés. 


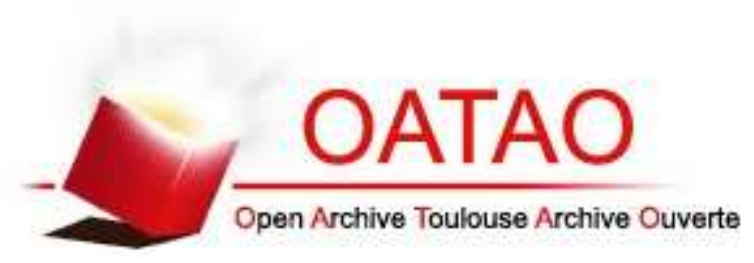

\section{Open Archive TOULOUSE Archive Ouverte (OATAO)}

OATAO is an open access repository that collects the work of Toulouse researchers and makes it freely available over the web where possible.

This is an author-deposited version published in : http://oatao.univ-toulouse.fr/ Eprints ID : 16789

To link to this article : DOI : 10.1088/2053-1583/3/2/025009

URL : http://dx.doi.org/10.1088/2053-1583/3/2/025009

To cite this version : Muzi, Laura and Mouchet, Florence and Cadarsi, Stéphanie and Janowska, Izabela and Russier, Julie and Ménard-Moyon, Cécilia and Risuleo, Gianfranco and Soula, Brigitte and Galibert, Anne-Marie and Flahaut, Emmanuel and Pinelli, Eric and Gauthier, Laury and Bianco, Alberto Examining the impact of multi-layer graphene using cellular and amphibian models. (2016) 2D Materials, vol. 3 ( $\mathrm{n}^{\circ} 2$ ). pp. 025009. ISSN 2053-1583

Any correspondence concerning this service should be sent to the repository administrator: staff-oatao@ listes-diff.inp-toulouse.fr 


\title{
Examining the impact of multi-layer graphene using cellular and amphibian models
}

\author{
Laura Muzi $^{1,2}$, Florence Mouchet ${ }^{3,4}$, Stéphanie Cadarsi $^{3,4}$, Izabela Janowska ${ }^{5}$, Julie Russier ${ }^{1}$, \\ Cécilia Ménard-Moyon ${ }^{1}$, Gianfranco Risuleo ${ }^{2}$, Brigitte Soula $^{6}$, Anne-Marie Galibert ${ }^{6}$, Emmanuel Flahaut ${ }^{6,7}$, \\ Eric Pinelli ${ }^{3,4}$, Laury Gauthier ${ }^{3,4}$ and Alberto Bianco ${ }^{1}$ \\ 1 CNRS, Institut de Biologie Moléculaire et Cellulaire, Laboratoire d'Immunopathologie et Chimie Thérapeutique, Strasbourg, France \\ 2 Dipartimento di Biologia e Biotecnologie, Sapienza Università di Roma, Italy \\ 3 Université de Toulouse, INP, UPS, EcoLab (Laboratoire Ecologie Fonctionnelle et Environnement), ENSAT, F-31326 Castanet Tolosan, \\ France \\ 4 CNRS, EcoLab (Laboratoire d'écologie fonctionnelle et environnement), F-31326 Castanet-Tolosan, France \\ 5 Institut de Chimie et Procédés pour l'Energie, l'Environnement et la Santé (ICPEES), F-67087 Strasbourg, France \\ 6 Université de Toulouse, INP, UPS, Institut Carnot CIRIMAT (Centre Inter-universitaire de Recherche et d'Ingénierie des Matériaux), \\ UMR CNRS 5085, F-31062 Toulouse cedex 9, France \\ 7 CNRS, Institut Carnot CIRIMAT, F-31062 Toulouse, France \\ E-mail: florence.mouchet@ensat.fr and a.bianco@ibmc-cnrs.unistra.fr
}

Keywords: few-layer graphene, cytotoxicity, macrophages, Xenopus larvae, mortality, genotoxicity

\begin{abstract}
In the last few years, graphene has been defined as the revolutionary material showing an incredible expansion in industrial applications. Different graphene forms have been applied in several contexts, spreading from energy technologies and electronics to food and agriculture technologies. Graphene showed promises also in the biomedical field. Hopeful results have been already obtained in diagnostic, drug delivery, tissue regeneration and photothermal cancer ablation. In view of the enormous development of graphene-based technologies, a careful assessment of its impact on health and environment is demanded. It is evident how investigating the graphene toxicity is of fundamental importance in the context of medical purposes. On the other hand, the nanomaterial present in the environment, likely to be generated all along the industrial life-cycle, may have harmful effects on living organisms. In the present work, an important contribution on the impact of multi-layer graphene (MLG) on health and environment is given by using a multifaceted approach. For the first purpose, the effect of the material on two mammalian cell models was assessed. Key cytotoxicity parameters were considered such as cell viability and inflammatory response induction. This was combined with an evaluation of MLG toxicity towards Xenopus laevis, used as both in vivo and environmental model organism.
\end{abstract}

\section{Introduction}

Graphene is one-atom-thick planar sheet of $\mathrm{sp}^{2}$ bonded carbon atoms that are densely packed in a honeycomb crystal lattice [1]. Graphene can display different forms which are conventionally combined under the so-called graphene family materials (GFMs). GFMs comprise few-layer graphene (FLG), multilayer graphene (MLG), graphene oxide (GO), reduced graphene oxide (rGO), graphene nanosheets, ultrafine graphite, graphene ribbons and graphene dots. FLG is constituted by $2-5$ graphene layers, while $\mathrm{GO}$ and $\mathrm{rGO}$ are normally composed of a single layer [2].

Despite being the basic structural element of other carbon allotropes, including carbon nanotubes and fullerenes, graphene history is quite recent. In fact, the research group, guided by Geim and Novoselov, reported for the first time a method for the isolation of single-layer graphene from graphite only in 2004 [3]. From its discovery, graphene has been the object of great industrial interest. Such success is due to its exceptional physico-chemical properties such as 
electronic, optical, thermal and mechanical which differ from the same material in bulk. Such properties can be tuned by choosing a particular type of graphene [4]. By taking advantage of its special characteristics, graphene-based nanotechnology represents nowadays an area of scientific research and industrial applications in full expansion [5, 6]. In fact, graphene was firstly exploited in material sciences and recently it showed to be a good candidate for the development of graphene-based electronics, photonics, composite materials, energy generation, energy storage and sensors [5-9]. Among all graphene forms, FLGs are easily produced in high yeld [10-13]. Low-cost procedures have also been described for the fabrication of large area films of FLG for electronics and opto-electronic applications [14].

This rapid increase in production and applications of graphene implies its potential release into the environment, especially into the aquatic compartment, which usually concentrates all kinds of contaminants [15]. For example, graphene release could occur from the use of commercial products containing the material, from its degradation during use and from the waste disposal of such products. The possible ecosystem risks induced by graphene were described by $\mathrm{Hu}$ and $\mathrm{Zhu}$ [15]. What is the environmental impact that can be expected after graphene introduction into the aquatic environment? The material would first interact with abiotic compounds such as natural organic matter and other molecules naturally present in waters (inorganic and organic macromolecules, colloidal particles, etc) but also with living organisms. Being important components of the ecosystem, the responses of aquatic organisms to graphene are particularly critical for ecosafety [15]. However, the existing knowledge of the potential toxicity of GFMs towards aquatic organisms is still poor. A recent review presents what is known on the toxicity of GFMs towards the aquatic environment [16]. Most of these works deal with the toxicity of GO and rGO on different aquatic organisms (bacteria, crustaceans, nematodes and fishes). Only a few studies report toxicity evaluation of single- and MLG. The general conclusion is that the impact of graphene against aquatic organisms may not be as high as carbon nanomaterials such as fullerenes and carbon nanotubes [16].

On the other hand, the possibility to isolate graphene flakes by repeated mechanical exfoliation of graphite, provides a material with a high degree of purity, making it suitable also for biomedical applications [17]. In addition, its high surface area allows for the conjugation with bioactive molecules such as DNA, proteins, peptides or small drug molecules [17]. Single- and FLG, demonstrated their potential in drug/ gene delivery, biosensing and imaging, antibacterial activity and tissue engineering [18-21]. It is evident how such applications are strictly dependent on graphene biocompatibility. In fact, its use for therapeutic purposes necessitates the demonstration of the absence of graphene-induced tissue damage or proinflammatory response. A large variety of studies can be found in literature reporting the toxicity findings on graphene in vitro and in vivo [22]. Despite this, the described results are sometimes in contrast. In fact, the biological response varies depending on graphene number of layers, lateral size, rigidity, hydrophobicity, dose administered and purity of the material. The use of diverse cellular models may also be responsible for paradoxical findings [22]. For this reason GFMs' toxicological profile is little understood and more studies concerning specific graphene types are required to avoid unexpected harmful effects on human health.

The current work is aimed at better elucidating the impact of graphene mainly consisting of 2-20 layers (MLG) on health and environment. The boundary between MLGs and graphite nanoplatelets is not sharp, so we decided to identify our material as MLGs even if the number of layers exceeds 10 (see [2] for a proposed nomenclature describing the different forms of graphene). For this purpose, two different mammalian cellular lines (human epithelial cells or murine macrophages) were used and key acute toxicity parameters such as cell viability and induction of proinflammatory response upon exposure to MLG were examined. The importance of MLG purification from adsorbed solvents was also taken into account. The in vitro preliminary studies were combined to the in vivo evaluation of MLG toxicity on the aquatic amphibian model organism Xenopus laevis. We used normalized exposure conditions [23] to assess larval mortality, growth inhibition and genotoxicity. On the other hand, the amphibian larvae represent also a relevant environmental model of aquatic organism to study the ecotoxicity of nanoparticle [24-27].

\section{Materials and methods}

\section{Materials}

MLGs were generated through a simple, fast and low cost production protocol with high yield previously described [10]. The flakes are made of sheets with different lateral size. This specific structure is useful for several applications (i.e. in catalysis, battery, supercapacitors and others, where the stabilization of metal particles are important) and it is related to the preparation process [28]. MLGs obtained by this method consist mainly of 2-20 sheets that, occasionally, can go up to fifty (see supporting figure S1). The thicker flakes were subsequently separated from the sample by a sedimentation process in toluene. The high purity sample (MLG1) was thermally treated to eliminate potentially adsorbed toluene between the graphene layers (MLG2) [29]. The lateral size of the MLG sheets ranged from 1.2 to $5.4 \mu \mathrm{m}$, with the average centered at $2.3 \mu \mathrm{m}$ (see supporting figure S2). MLG dispersions were prepared freshly prior to each cellular exposure by sonicating the material in cell 
culture medium containing $10 \%$ of fetal bovine serum (FBS), at a concentration of $100 \mu \mathrm{g} \mathrm{ml}^{-1}$. The samples were diluted to the proper concentration before use.

\section{Transmission electron microscopy}

The morphology and number of MLG flakes were examined by high-resolution transmission electron microscopy on a Topcon 002B-UHR microscope working with an accelerated voltage of $200 \mathrm{kV}$ and a point-to-point resolution of $0.17 \mathrm{~nm}$. Prior to the analysis, the sample was ultrasonically dispersed in ethanol during $5 \mathrm{~min}$ and a drop of the suspension was deposited onto a perforated carbon membrane covered copper grid.

\section{Cell cultures}

HeLa cells (human tumor-derived epithelial cells) and RAW 264.7 cells (murine transformed macrophages) were purchased from ATCC (VA, USA). Both cell lines were cultured in RPMI 1640 medium supplemented with $10 \%$ heat inactivated FBS and $100 \mathrm{U} \mathrm{ml}^{-1}$ gentamycin (with the addition of $50 \mu \mathrm{M} \beta$-mercaptoethanol and $20 \mathrm{mM}$ HEPES for RAW 264.7 macrophages). Cells were maintained at $37^{\circ} \mathrm{C}$ in humidified air containing 5\% $\mathrm{CO}_{2}$. Media and supplements were purchased from Lonza. When confluency reached 70\%-80\%, HeLa or RAW 264.7 cells were washed with phosphate-buffered saline (PBS), detached with trypsin or with SE buffer (PBS containing $2 \mathrm{mM}$ EDTA and 2\% FBS), respectively, and subcultured each 2-3 d. Before MLG exposure, cells were detached, counted and reseeded in proper well size and density (see each particular experiment for details) and allowed to adhere overnight.

\section{Flow cytometry}

For cell viability experiments, HeLa or RAW 264.7 cells were seeded into 96-well culture plates at a density of $1 \times 10^{5}$ cells per well and allowed to adhere overnight. Cells were exposed to different concentrations (ranging from 1 to $100 \mu \mathrm{g} \mathrm{ml}^{-1}$ ) of MLG1 or MLG2 for $24 \mathrm{~h}$. DMSO (20\%) was used as positive control for cellular death. After incubation, RAW 264.7 supernatants were collected for further investigations while cells were harvested and stained with FITC-Annexin V (AnnV; BD Pharmingen 556419) and propidium iodide (PI, $0.2 \mu \mathrm{g} \mathrm{ml}^{-1}$; SigmaAldrich) in a calcium containing buffer. The percentage of live (AnnV negative and PI negative), early apoptotic (AnnV positive and PI negative) and late apoptotic/necrotic (AnnV positive and PI positive plus AnnV negative and PI positive) cells was determined by acquiring at least 50000 events using a Gallios flow cytometer (Beckman Coulter, Villepinte, France) and analyzing the data with FlowJo software.

For the analysis of cell activation, after incubation with MLG1 or MLG2, RAW 264.7 cells were harvested and stained with PE-Rat anti-Mouse CD86 antibodies
(Clone GL1, BD Pharmingen 553692). Lipopolysaccharide (LPS, $1 \mu \mathrm{g} \mathrm{ml}^{-1}$ ) in combination with interferon $\gamma$ (IFN $\gamma, 1 \mathrm{ng} \mathrm{ml}^{-1}$ ) was used as positive control. The macrophage-associated CD86 fluorescence intensity was determined by acquiring at least 50000 events using the Gallios flow cytometer and analyzing the data on the live cell gated population with FlowJo software.

The flow cytometry results are a summary of the data from at least five separate experiments run in triplicate for each cell line. Data are presented \pm SEM. Statistical analyzes were performed using a two-way ANOVA test followed by Bonferroni's post-test. All $p$ values $<0.05$ were considered significant.

\section{ELISA}

The concentration of interleukine 6 (IL6) and tumor necrosis factor alpha (TNF $\alpha$ ) in RAW 264.7 cell supernatants were examined by a double sandwich ELISA. LPS $\left(1 \mu \mathrm{g} \mathrm{ml}^{-1}\right)$ in combination with IFN $\gamma$ $\left(1 \mathrm{ng} \mathrm{ml}^{-1}\right)$ was used as positive control. Polyvinyl microtiter plates (Falcon) were coated overnight at $4{ }^{\circ} \mathrm{C}$ with $50 \mu \mathrm{l}$ per well of purified Rat Anti-Mouse IL6 (BD Pharmingen 554400) or purified Hamster Anti-Mouse/Rat TNF $\alpha$ (BD Pharmingen 557516) antibodies diluted in $0.05 \mathrm{M}$ carbonate buffer, $\mathrm{pH}$ 9.6. Non-specific sites were saturated with $100 \mu \mathrm{l}$ per well of PBS containing $10 \%$ FBS for $1 \mathrm{~h}$ at $37^{\circ} \mathrm{C} .50 \mu \mathrm{l}$ per well of culture supernatants or Recombinant Mouse IL6 (BD Pharmingen 554582) or Recombinant Mouse TNF (BD Pharmingen 554589) diluted in PBS-10\% FBS, were added for $2 \mathrm{~h}$ at $37^{\circ} \mathrm{C} .50 \mu \mathrm{l}$ per well of secondary Biotin Rat Anti-Mouse IL6 (BD Pharmingen 554402) or Biotin Rabbit Anti-Rat/Mouse TNF (BD Pharmingen 557432) diluted in PBS-10\% FBS, were added for $1 \mathrm{~h}$ at $37^{\circ} \mathrm{C} .50 \mu \mathrm{l}$ of streptavidin conjugated to horseradish peroxidase diluted in PBS $10 \%$ FBS were added per well. Plates were washed after each step with PBS containing $0.05 \%$ of tween 20 (PBS-T). After $30 \mathrm{~min}$ incubation at $37^{\circ} \mathrm{C}$, plates were washed extensively and the enzymatic reaction was visualized by adding $75 \mu \mathrm{l}$ per well of $3,3^{\prime}, 5,5^{\prime}$ tetramethylbenzidine diluted in $0.1 \mathrm{M}$ citrate buffer $(\mathrm{pH} 5)$ in the presence of $\mathrm{H}_{2} \mathrm{O}_{2}$. The resulting absorbance was measured at $450 \mathrm{~nm}$ after the reaction was stopped with $25 \mu \mathrm{l}$ per well of $\mathrm{HCl} 1 \mathrm{~N}$. Statistical analyzes were performed as previously described in the flow cytometry section.

\section{Xenopus breeding and maintenance}

Eggs were obtained at the Ecolab laboratory resulting from the mating of two Xenopus adults following the hormonal injection of 50 IU of PMSG 500 (Pregnant Mare's Serum Gonadotrophin, Intervet, France) for the male and 750 IU of HCG (Human Chorionic Gonadotropin, Organon, France) for the female. Viable eggs were maintained in aquariums containing normal tap water filtered through active charcoal at 
$20{ }^{\circ} \mathrm{C}-22{ }^{\circ} \mathrm{C}$ until they reached the development stage appropriate for experimentation. The larvae were fed with dehydrated aquarium fish food every day (Tetraphyll $^{\circledR}$,Zolux, France).

\section{Xenopus exposure conditions}

The exposure was performed according to the International Standard 21427-1 [23] in semi-static exposure conditions with larvae at stage 50 [30]. The larvae were taken from the same laying to reduce inter-individual variability. They were exposed for $12 \mathrm{~d}$ in groups of 20 animals in crystallizing dishes containing either MLG concentrations $\left(0,0.1,1,10\right.$ and $\left.50 \mathrm{mg} \mathrm{l}^{-1}\right)$ in reconstituted water (RW, i.e., chlorine-free water supplemented with nutritive salts, as described in ISO 21427-1 (294 mg l ${ }^{-1} \quad \mathrm{CaCl}_{2} \cdot 2 \mathrm{H}_{2} \mathrm{O}, 123.25 \mathrm{mg} \mathrm{l}^{-1}$ $\mathrm{MgSO}_{4} \cdot 7 \mathrm{H}_{2} \mathrm{O}, 64.75 \mathrm{mgl}^{-1} \mathrm{NaHCO}_{3}, 5.75 \mathrm{mgl}^{-1}$ $\mathrm{KCl}, \mathrm{pH} 7$ ) or control media, i.e. $\mathrm{RW}$ without $\mathrm{MLG}$ (negative control: NC and positive control: PC). The PC was performed to check the responsiveness of the amphibian larvae using the addition of cyclophosphamide (CP 6055-19-2, Sigma, France) at $20 \mathrm{mg} \mathrm{l}^{-1}$ to RW. 2 stock suspensions at 1 and $10 \mathrm{gl}^{-1}$ were prepared by sonicating the required amount of dry MLG powder in the required amount of deionized water. For the $10 \mathrm{gl}^{-1}$ (respectively $1 \mathrm{gl}^{-1}$ ) stock suspension, $1.5 \mathrm{~g}$ (respectively $0.155 \mathrm{~g}$ ) of dry powder was weighed and introduced in a $200 \mathrm{ml}$ glass flask. $150 \mathrm{ml}$ (respectively $155 \mathrm{ml}$ ) of ultra-pure water were added, and the mixture was tip-sonicated (Vibra Cell $75042,20 \mathrm{kHz}, 500 \mathrm{~W}, 30 \%$ amplitude with $5 \mathrm{~s}$ on $/ 5 \mathrm{~s}$ off pulses) for $30 \mathrm{~min}$. Glass flasks were placed in a crystallizing dish containing ice-cooled water during the tip sonication in order to prevent overheating during this step. Twelve glass tubes containing $100 \mathrm{mg}$ or $20 \mathrm{mg}$ in $20 \mathrm{ml}$ of ultra-pure water were separately prepared from a stock suspension of $10 \mathrm{gl}^{-1}$ $(1469 \mathrm{mg} / 146.9 \mathrm{ml})$ by sampling respectively $10 \mathrm{ml}$ or $2 \mathrm{ml}$ after $10 \mathrm{~min}$ of ultrasonication (Bioblock T570, $35 \mathrm{kHz}, 160 \mathrm{~W}$ ). Twelve other tubes containing $2 \mathrm{mg}$ or $0.2 \mathrm{mg}$ in $20 \mathrm{ml}$ of ultra-pure water were separately prepared from a second stock suspension of $1 \mathrm{gl}^{-1}$ ( $40 \mathrm{mg} / 40 \mathrm{ml}$ ) by sampling respectively $2 \mathrm{ml}$ or $0.2 \mathrm{ml}$ after 10 min of ultrasonication. Tube content was daily sonicated during $5 \mathrm{~min}$ and transferred into the appropriate crystallizing dishes before simply adjusting the volume to 21 with RW. Larvae are then reintegrated in appropriate dishes and fed (Tetraphyll ${ }^{\circledR}$, Zolux, France). The larvae were submitted to a natural light-dark cycle at $22.0 \pm 0.5^{\circ} \mathrm{C}$ during the 12 d of exposure.

\section{Xenopus toxicity evaluation}

Mortality of larvae exposed to MLG was examined for $12 \mathrm{~d}$ according to the standardized recommendations [23] by visual inspection. The accepted level of significance is $20 \%$ mortality, corresponding to 4 out of 20 larvae dead, excluding the idea that mortality could be manually caused during media changes.

Growth inhibition was evaluated by measuring the size of each larva $(n=20)$ at the beginning of the exposure $\left(t_{0}\right)$ and at the end of the exposure $\left(t_{12}\right)$ using the Mesurim image analysis software [31]. Statistical analyzes were performed using SigmaStat 3.1 according to non parametric tests (Kruskal-Wallis followed by Dunn's or Dunnet's test and Mann-Whitney test) described in previous studies [24]. Graphic representations are proposed, based on the growth rate calculated, as described in these previous studies.

For the micronuclei test (MNT), at the end of exposure, a blood sample was obtained from each anaesthetized larva (MS222, Sandoz, France). Technical procedures are well described on the standardized recommendation fascicle [23]. The number of erythrocytes that contained one micronucleus or more (micronucleated erythrocytes, MNE) was determined in a total sample of 1000 erythrocytes per larva (MNE $\%$ ). Based on median values and quartiles [32], the number of micronucleated erythrocytes per thousand, MNE \%o is presented with their $95 \%$ confidence limits expressed by the median $\pm 1.57 \times$ IQR inter-quartile range (upper quartile-lower quartile) $/ \sqrt{ } n$. The difference between the theoretical medians of the test groups and the theoretical median of the negative control group is significant to within $95 \%$ certainty if there is no overlap.

After blood puncturing, the general aspect of the larvae exposed to MLG was visually compared to that of NC group under the binocular. After dissection of some larvae of each group, their guts were then observed under the binocular (magnification $\times 15$ ) to observe the presence or absence of MLG.

\section{Results and discussion}

\section{Toxicity on cellular models}

Inspecting nanomaterial acute toxicity on in vitro cellular models is an important first step for an appropriate assessment of their biocompatibility. For this purpose, tumor-derived epithelial cells (HeLa) were selected as valid human model to test the possible cytotoxicity of two MLG samples. It is important to point out that this cellular type represents a nonphagocytic model. The tests were performed also on a macrophage cell line (RAW 264.7). The latter was chosen as an important immune cell model, in order to compare the effects of MLG on the two cell lines and to further investigate the possible proinflammatory response triggered by MLGs. As a matter of fact, macrophages are one of the major actors during the primary immune response against infectious organisms and external materials, being able to phagocyte such entities and trigger secondary immune reactions [33]. The importance of MLG purification on cytotoxicity was also taken into account. For this purpose, 

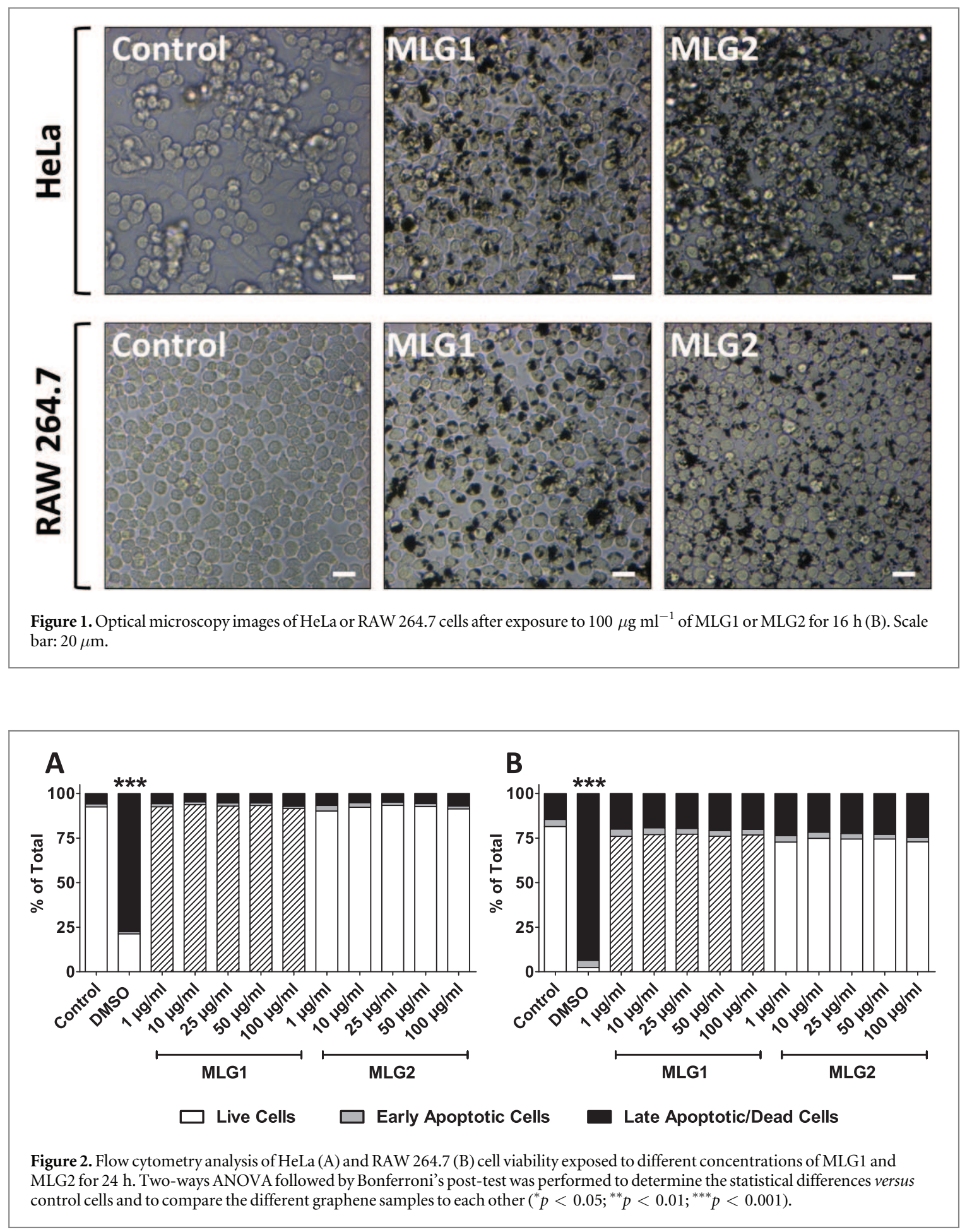

two different MLG samples were tested: MLG1, corresponding to the starting MLG batch and MLG2, consisting in MLG1 thermally treated to eliminate residual toluene molecules potentially adsorbed and entrapped between the graphene layers.

Cell viability was the first parameter that we analyzed. Both cellular models were exposed to increasing concentrations of MLG1 or MLG2 for $24 \mathrm{~h}$. It is important to point out that the dispersion of both MLG samples in cell culture medium was not optimal. Optical microscopy images of the treated cells were taken after about $16 \mathrm{~h}$. It was soon clear how the degree of MLG aggregation was very high (figure 1). On the other hand, it was also possible to observe that MLG black spots often colocalized with the cells suggesting an interaction with the cell membranes. However, the general cellular morphology seemed comparable to control cells. At the end of the incubation, the cell viability was determined by flow cytometry upon AnnV/PI staining. Data show that HeLa cells tolerated very well both MLG samples as no reduction of cell viability was observed (figure 2(A)). On the contrary, they were dramatically affected by control DMSO. A similar situation was monitored in 


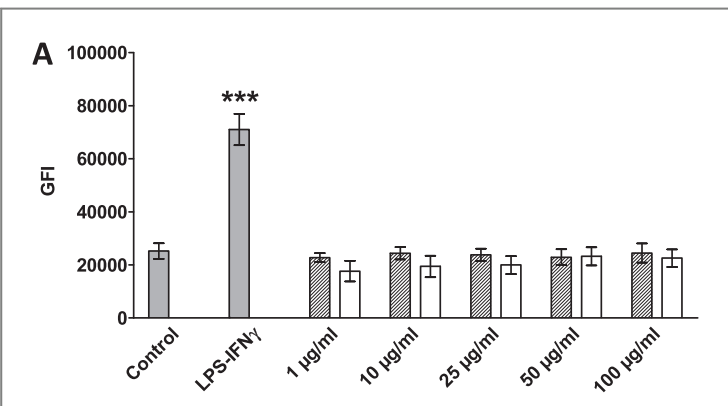

B

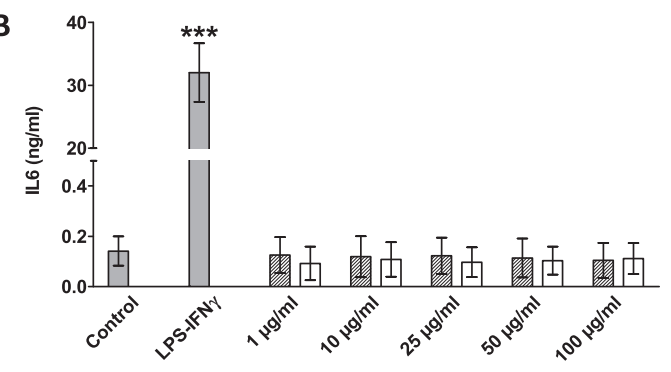

C

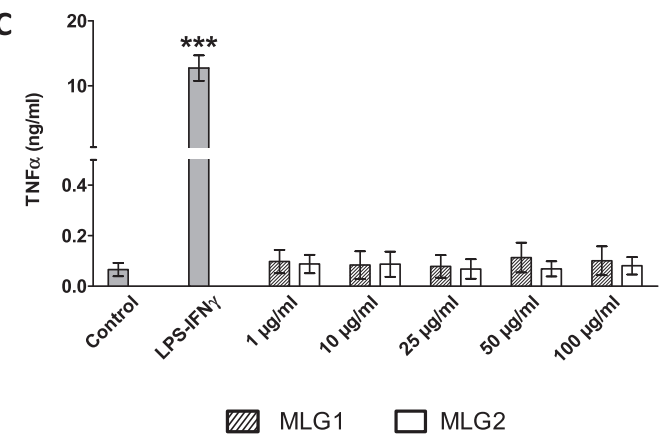

Figure 3. Flow cytometry analysis of CD86 expression (A) and cytokine production (IL6 panel (B), TNF $\alpha$ panel (C)) in RAW 264.7 cells exposed to different concentrations of MLG1 and MLG2 for $24 \mathrm{~h}$. GFI = geomean fluorescence intensity. Twoways ANOVA followed by Bonferroni's post-test was performed to determine the statistical differences versus control cells and to compare the two graphene samples to each other $\left({ }^{*} p<0.05 ;{ }^{* *} p<0.01 ;{ }^{* * *} p<0.001\right)$.

the case of RAW 264.7 cells. In fact, MLG1 and MLG2 induced only a slight, but not significant, decrease of cell viability (of about $5 \%$ ) in the macrophage cell line (figure 2(B)). No differences between the two MLG samples were evidenced.

Investigating the immunosafety of a new material is also of fundamental importance in the context of biomedical applications. In fact, the immune cells of the body may be activated by the external molecules and elicit a harmful inflammatory response [34]. To assess this second important parameter, RAW 264.7 macrophages were incubated with increasing concentrations of MLG1 or MLG2, as previously described, and the expression of the macrophage activation marker CD86 was analyzed by flow cytometry. The culture supernatants were also collected to evaluate the levels of two proinflammatory cytokines, (IL6 and TNF $\alpha$ ) by ELISA. The results show that the levels of CD86 and cytokines were comparable to control cells, indicating that our MLG samples did not induce any proinflammatory response in RAW 264.7 macrophages (figure 3). On the contrary cell activation and cytokine levels increased when the cells were treated with LPS/IFN $\gamma$ thus validating the test.

Our data on cell viability and cell activation suggest that MLG does not elicit any cytotoxic effects. However, such result is in contrast with some previous findings [22, 35]. To give a few examples, an early study reported the induction of high oxidative stress and caspase- 3 activation (indicating apoptotic process) in neuronal rat cells exposed to pristine graphene (composed mainly of 3-5 layers) [36]. Another group showed that 3-4 layers GO can trigger cell cycle alterations, apoptosis, and oxidative stress in osteoblast and macrophage cell models [37]. However, graphene cytotoxicity depends on several factors such as graphene type (in terms of functionalities, number of layers and lateral size), graphene impurities and cell type tested, as it is reported in different reviews [22, 35]. Nevertheless, we can make a hypothesis to explain the discrepancy between our and literature data on graphene cytotoxicity. Our MLG samples are composed of several graphene sheets (2-20), while the majority of the works utilize single-layer or FLG (pristine or oxidized). Studies mainly focused on the graphene sheet lateral size, rather than the number of layers [38, 39]. The number of graphene layers is instead a very important parameter we have to consider because it determines specific surface area and bending stiffness [40]. In particular, the specific area is inversely proportional to the number of graphene layers. As a consequence, surface phenomena such as physical adsorption of molecules or catalytic chemical reactions, occurring within the biological environment, are reduced in our case. Since such events may be responsible for adverse biological reactions [40], this could explain the absence of cytotoxicity observed in our system. Furthermore, in our conditions, MLG dispersions were not optimal, as evidenced by the strong presence of aggregates (figure 1), thus decreasing even more the phenomena. Another hypothesis can also be made and concerns the material internalization. We assume that the stiffness of our MLG samples is substantially high [40]. Despite being phagocytic cells, macrophages may fail to internalize a material with this rigidity and even more large MLG aggregates, thus explaining the negligible effect on their cell viability and activation. The passive diffusion of the material trough the plasma membrane, previously reported $[39,41]$, might also be negatively affected by MLG high stiffness. However, it has been reported that microsized graphene (lateral size comprised between 0.5 and $25 \mu \mathrm{m}$ ) composed of 4-25 layers, can be internalized by macrophages [41]. The authors hypothesize that the interaction between the hydrophobic basal surface of graphene microsheets with the inner hydrophobic region of the plasma membrane promoted the cellular uptake. Despite a lower lateral size, the aggregation status of our MLGs is likely sufficient to support the hypothesis of a lack of internalization. In the same 
work, it has been also proposed that graphene microsheets, once internalized by macrophages, may be recognized as damage-associated molecular patterns that nonspecifically activate the innate immune response [42]. In our case, MLG hydrophobic surface area (average lateral size $2.3 \mu \mathrm{m}$ and number of layers 2-20) is low and this represents another possible reason to explain the absence of proinflammatory reaction in RAW 264.7 macrophages. The importance of the material shape and size on macrophage inflammatory response was reported by us and other groups $[38,39]$. Another previous study was focused on the cellular impact of GO sheets having different lateral sizes. The authors showed that only GO samples with sheets of about $2 \mu \mathrm{m}$ triggered strong primary macrophage activation, contrarily to sheets of about $300 \mathrm{~nm}$ [38]. On the contrary, we have previously shown how, the smaller the GO lateral size, the higher the material internalization and the higher the proinflammatory effect was present, in both human and murine primary macrophages [39]. The cellular uptake of GO with the higher lateral size $(1.32 \mu \mathrm{m}$ in average) was less efficient compared to sheets with average lateral sizes of 0.27 and $0.13 \mu \mathrm{m}$, respectively. The large GO sample was also less cytotoxic than the two GOs in the nanoscale range towards macrophages. These previous results are fully consistent with the data obtained in this work, as our MLGs have an average lateral size of $2.3 \mu \mathrm{m}$.

These observations give a further demonstration that several parameters influence graphene toxicity. Despite we were not able to precisely evaluate the relative contribution of each parameter on MLG cytotoxicity, we can hypothesize that the relatively high number of layers and lateral size of MLGs combined with a certain degree of aggregation have a synergistic effect in determining the absence of toxicity in our cellular models. This is likely to be due to a poor material uptake and to the reduced hydrophobic surface area (related to the layer number of MLGs) which reduce surface adsorption phenomena considered responsible of promoting inflammatory responses [40].

\section{Toxicity on Xenopus larvae}

The highly pure MLG sample was further tested on $X$. laevis. In this work, the amphibian was used for a double scope. First, it is a valid model to further assess the in vivo impact of MLGs, giving more insights on the overall toxicity of the material. Second, the in vivo results give also information about the ecotoxicity of MLGs as X. laevis is a relevant environmental model of aquatic organism to study the ecotoxicity of nanoparticle [24-27].

The first parameter that we considered was larval survival after contact with the material, as index of acute toxicity. The results show no mortality in larvae exposed in presence of MLGs, whatever the concentration.
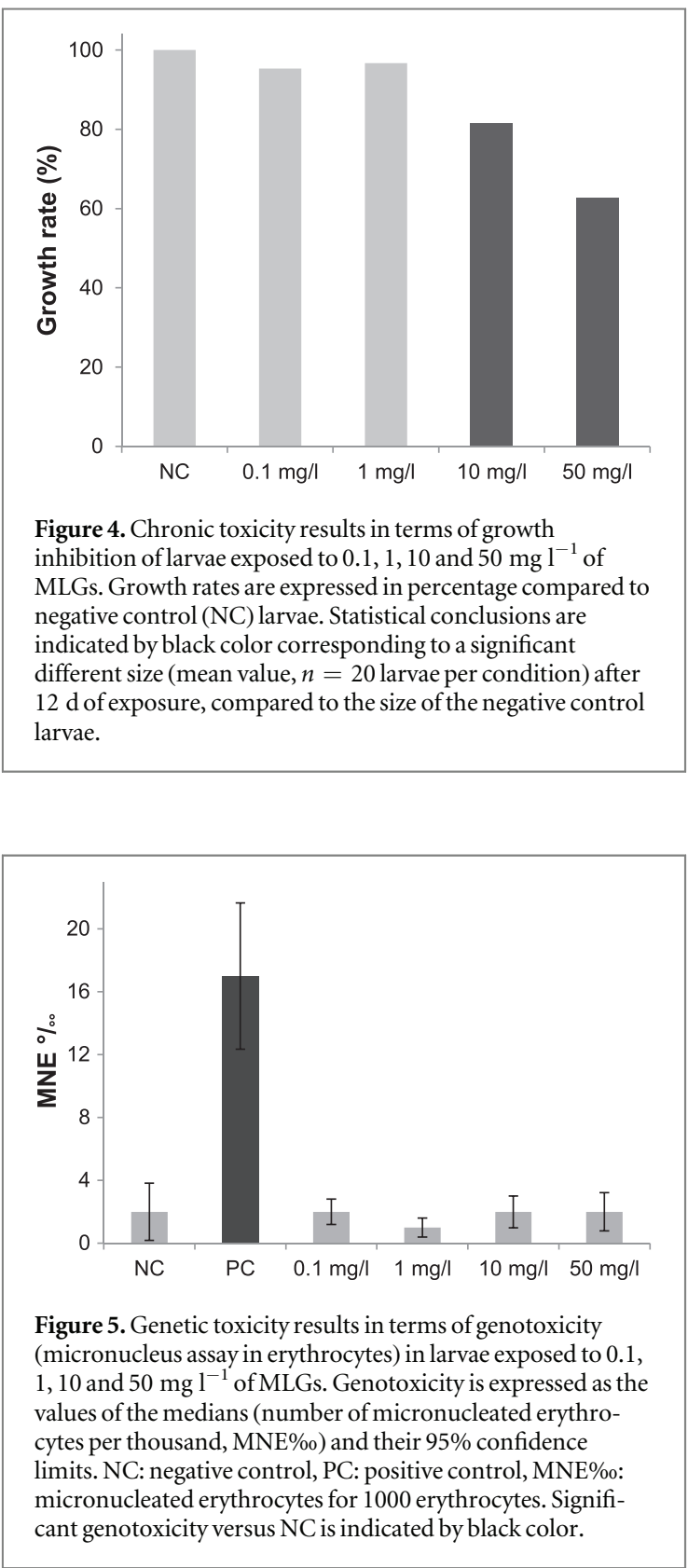

As second factor, we analyzed the variation of larval size, representing an indication of chronic toxicity, in response to MLGs. The measurements of the larval size show that larvae exposed in presence of 10 and $50 \mathrm{mg} \mathrm{l}^{-1}$ of the material have significantly reduced size compared to the NC. However, no significant reduced size was observed at 0.1 and $1 \mathrm{mgl}^{-1}$ (figure 4).

The assessment of whether MLGs were able to induce damage in the genetic materials of living organisms, such as DNA mutations and/or chromosomal aberrations, is also very important. Micronucleus induction is a valid cytogenetic biomarkers used for measuring the genotoxic potential of agents. Figure 5 shows that larvae exposed to PC have significantly higher MNE \%o as compared to the NC group $(17 \pm 4.56$ versus $2 \pm 1.82)$, validating the MNT results. No genotoxicity via micronucleus induction in 


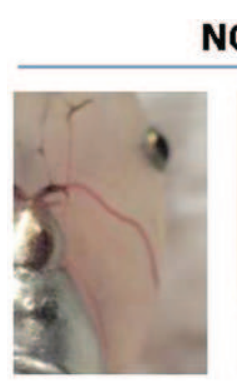

\section{NC}

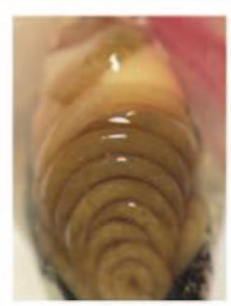

MLG $0.1 \mathrm{mg} / \mathrm{l}$

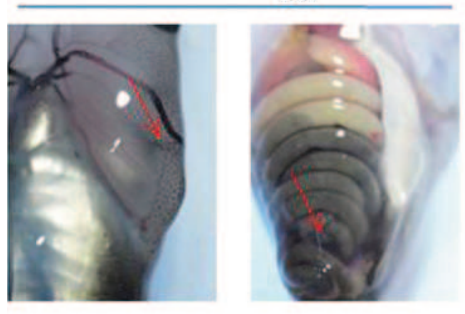

MLG $1 \mathrm{mg} / \mathrm{l}$

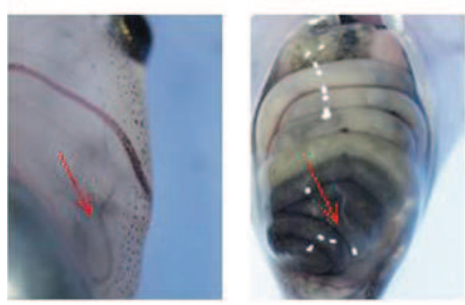

MLG $10 \mathrm{mg} / \mathrm{l}$

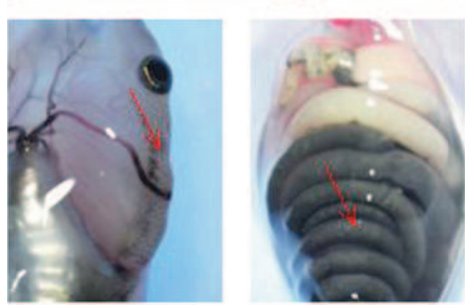

MLG $50 \mathrm{mg} / \mathrm{l}$

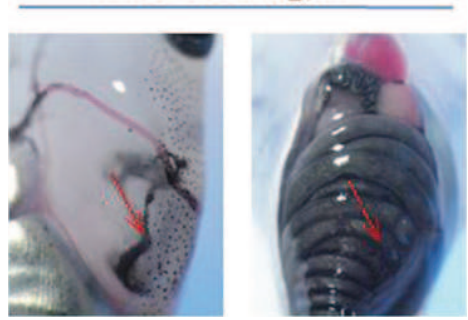

Figure 6. Macro observation of Xenopus larvae exposed to 0.1, 1,10 and $50 \mathrm{mg} \mathrm{l}^{-1}$ of MLGs in comparison to NC larvae. Red arrows indicate the suspicion of the presence of MLGs in gills and in the intestines.

erythrocytes of $X$. larvae was observed, whatever the concentration of MLGs. Indeed, the median values of MNE \%o were $2 \pm 0.81,1 \pm 0.61,2 \pm 1.01$ and $2 \pm 1.22$ for respectively $0.1,1,10$ and $50 \mathrm{mg} \mathrm{l}^{-1}$.

$X$. larvae exposed during $12 \mathrm{~d}$ to MLGs in RW displayed a particular visual aspect compared to NC larvae (figure 6). Black agglomerates, supposed to be

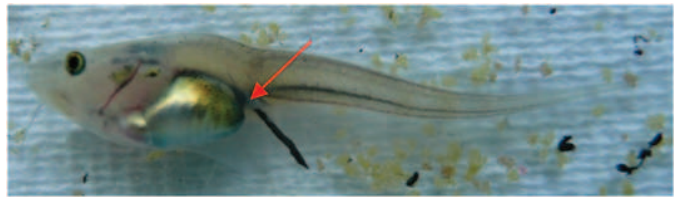

Figure 7. Larval excretion of the suspected MLGs of the media exposure by the anal $\left(50 \mathrm{mg} \mathrm{l}^{-1}\right)$. Red arrow indicates the anal region and the excretion of the agglomerated MLGs into the excrements.

MLG, are increasingly observed in gills and intestines when the concentration of MLGs in media exposure increases. In addition, larval excretion of MLGs agglomerated with excrements is easily observed, for example in figure 7 , after exposure to $50 \mathrm{mgl}^{-1}$ of MLGs.

Results on X. larvae show that MLG is substantially not toxic for this aquatic species. Such data are consistent with those found in the current literature on graphene ecotoxicity. Zanni et al showed that graphite nanoplates did not induce oxidative stress in the bacteria Pseudomonas aeruginosa, but $70 \%$ of bacterial viability was lost after $5 \mathrm{~h}$ of exposure to $250 \mathrm{mg} \mathrm{l}^{-1}$ of the material [43]. They also showed no lifespan difference after exposing the nematode Caenorhabditis elegans to $250 \mathrm{mgl}^{-1}$ of nanoplates, although they observed the distribution of the material along the body of the nematode. In another study, the authors calculated the half maximal effective concentration $\left(\mathrm{EC}_{50}\right)$ values of 1.92 and $1.42 \mathrm{mg} \mathrm{l}^{-1}$ after $30 \mathrm{~min}$ of exposure of the bacteria Vibrio fischeri to monolayer graphene and graphene nanopowder, respectively [44]. They also calculated $\mathrm{EC}_{50}$ of 1.14 and $2.25 \mathrm{mg} \mathrm{l}^{-1}$ after $72 \mathrm{~h}$ of exposure of the algae Dunaliella tertiolecta to monolayer graphene and graphene nanopowder, respectively. No acute toxicity against the crustacean Artemia salina was detected although aggregation of graphene into the gut was evidenced [44]. Guo and collaborators showed ${ }^{14} \mathrm{C}$-labeled graphene content in the body of the crustacean Daphnia magna ( $1 \%$ of the organism dry mass) after $24 \mathrm{~h}$ of exposure to $250 \mu \mathrm{g} \mathrm{l}^{-1}$ of the material [45]. Another work reported the distribution of $100 \mathrm{mg} \mathrm{l}^{-1}$ of multi-functional graphene from head to tail of the embryos of the fish Danio rerio. However, the material did not induce abnormalities in the organisms [46].

In our case, only the $X$. larvae growth rate seemed to be affected by the material to some extent. Indeed, the larval size was decreased by about $40 \%$ only upon exposure to the highest concentration of MLGs $\left(50 \mathrm{mgl}^{-1}\right)$. This profile response is usually observed in X. larvae exposed under the same conditions to carbon nanotubes [24, 25]. Several hypotheses have already been proposed to explain this phenomenon. For instance, carbon-based nanomaterial uptake may lead to digestive (gut) and respiratory (gills) obstruction causing some exchange gas dysfunctions. A 
competition between nutrients and carbon based nanomaterials might be another explanation. Carbonbased nanomaterials, quickly sediment into the water column at the bottom of experimental dishes forming agglomerates with food and excreta during the exposure. When larvae browse at the bottom of the experimental dishes, massive larval uptake of the agglomerates occurs, thus leading to respiratory and/ or intestinal clogging.

On the other hand, MLG did not induce larval mortality and genotoxicity, at any of the concentrations tested. Such data support the results obtained on the human epithelial cells (HeLa) and on murine macrophages (RAW 264.7). MLG agglomerates could be then excreted without triggering any genetic toxicity towards the amphibian. One can speculate that, also in vivo, our particular MLGs cannot be internalized by the cells they encounter. Such cells are mainly represented by the epithelial cells of the respiratory and digestive tracts, which are the main way of entry in our case. Consequently, MLG may not be able to reach other body compartments such as the blood flow, thus explaining also the absence of genotoxicity towards erythrocytes. In fact, our previous studies using transmission electron microscopy, Raman spectroscopy and histology could not clearly evidence the translocation of carbon nanotubes through the intestinal barrier in Xenopus [24, 47].

To conclude, in this multifaceted work, MLGs composed of 2-20 graphene layers, were tested on two mammalian cell models and on X. laevis as an important in vivo and environmental model organism. MLG showed to be substantially not toxic towards our cellular models and $X$. larvae. We hypothesize that the absence of harmful effects both in the in vitro and in vivo models can be ascribed to different factors. The first reason might be represented by the failure in MLG cellular internalization, due to the particular size-related characteristics of our material [40], and to its poor dispersibility in aqueous media. It is conceivable that cells may encounter more difficulty in internalizing a material aggregated by endocytosis/phagocytosis, thus explaining the absence of toxicity. On the other hand, the specific surface area of our MLG is low and less exposed to the surrounding biological environment, compared to monolayer or FLG-based materials [40]. For this reason, the material might not be recognized by macrophages as a hydrophobic damage-associated molecular pattern, able to activate the innate immune response [42]. In addition, surface phenomena such as physical adsorption of molecules or catalytic chemical reactions, occurring within the biological environment, are reduced in our case. Since such events may be responsible for adverse biological reactions [40], this could also explain the absence of cytotoxicity observed in our system.

This study gives an important contribution on the impact of MLG on health and environment and further evidences the importance of graphene size, shape and dispersibility on its biological impact and on the synergistic effects that different parameters might have in triggering toxic effects.

\section{Acknowledgments}

This work was supported by the Centre National de la Recherche Scientifique (CNRS), by the Agence Nationale de la Recherche (ANR): LabEx project Chemistry of Complex Systems (ANR-10-LABX-0026_CSC), and by the International Center for Frontier Research in Chemistry (icFRC). The authors gratefully acknowledge financial support from EU FP7-ICT-2013-FET-F GRAPHENE Flagship project (no. 604391) and from the CNRS Program 'Défi G3N' 2012.

\section{References}

[1] Geim A K and Novoselov K S 2007 The rise of graphene Nat. Mater. 6 183-91

[2] Bianco A et al 2013 All in the graphene family-a recommended nomenclature for two-dimensional carbon materials Carbon 65 1-6

[3] Novoselov K S, Geim A K, Morozov S V, Jiang D, Zhang Y, Dubonos S V, Grigorieva IV and Firsov A A 2004 Electric field effect in atomically thin carbon films Science 306 666-9

[4] Huang X, Yin Z, Wu S, Qi X, He Q, Zhang Q, Yan Q, Boey F and Zhang H 2011 Graphene-based materials: synthesis, characterization, properties, and applications Small 7 1876-902

[5] Novoselov K S, Fal'ko V I, Colombo L, Gellert P R, Schwab M G and Kim K 2012 A roadmap for graphene Nature 490 192-200

[6] Ferrari A C et al 2015 Science and technology roadmap for graphene, related two-dimensional crystals, and hybrid systems Nanoscale 7 4598-810

[7] Kavan L, Yum J H and Gratzel M 2011 Optically transparent cathode for dye-sensitized solar cells based on graphene nanoplatelets ACS Nano 5 165-72

[8] Pumera M 2009 Electrochemistry of graphene: new horizons for sensing and energy storage Chem. Rec. 9 211-23

[9] Keele G P, O’Neill A, McEvoy N, Peltekis N, Colemanac J N and Duesberg G S 2010 Electrochemical ascorbic acid sensor based on DMF-exfoliated graphene J. Mater. Chem. 20 7864-9

[10] Janowska I, Vigneron F, Bégin D, Ersen O, Bernhardt P, Romero T, Ledoux M J and Pham-Huu C 2012 Mechanical thinning to make few-layer graphene from pencil lead Carbon 503106-10

[11] Liu X, Zheng M, Xiao K, Xiao Y, He C, Dong H, Lei B and Liu Y 2014 Simple, green and high-yield production of single- or few-layer graphene by hydrothermal exfoliation of graphite Nanoscale $64598-603$

[12] Paton K R et al 2014 Scalable production of large quantities of defect-free few-layer graphene by shear exfoliation in liquids Nat. Mater. 13 624-30

[13] Wu L, Li W, Li P, Liao S, Qiu S, Chen M, Guo Y, Li Q, Zhu C and Liu L 2014 Powder, paper and foam of few-layer graphene prepared in high yield by electrochemical intercalation exfoliation of expanded graphite Small 10 1421-9

[14] Reina A, Jia X, Ho J, Nezich D, Son H, Bulovic V Dresselhaus M S and Kong J 2009 Large area, few-layer graphene films on arbitrary substrates by chemical vapor deposition Nano Lett. 930-5

[15] Hu X and Zhou Q 2013 Health and ecosystem risks of graphene Chem. Rev. 113 3815-35 
[16] Zhao J, Wang Z, White J C and Xing B 2014 Graphene in the aquatic environment: adsorption, dispersion, toxicity and transformation Environ. Sci. Technol. 48 9995-10009

[17] Spinato C, Ménard-Moyon C and Bianco A 2014 Chemical functionalization of graphene for biomedical applications Functionalization of Graphene ed V Georgakilas (Weinnheim: Wiley-VHC) pp 95-139

[18] Shen H, Zhang L, Liu M and Zhan Z 2012 Biomedical applications of graphene Theranostics 2 283-94

[19] Liu J, Cui L and Losic D 2013 Graphene and graphene oxide as new nanocarriers for drug delivery applications Acta Biomater. 99243-57

[20] Goenka S, Sant V and Sant S 2014 Graphene-based nanomaterials for drug delivery and tissue engineering J. Control. Release $17375-88$

[21] Shi S, Chen F, Ehlerding E B and Cai W 2014 Surface engineering of graphene-based nanomaterials for biomedical applications Bioconjug. Chem. 25 1609-19

[22] Bianco A 2013 Graphene: safe or toxic? The two faces of the medal Angew. Chem., Int. Ed. Engl. 52 4986-97

[23] ISO 21427-12006 Water quality -Evaluation of Genotoxicity by Measurement of the Induction of Micronuclei-Part 1: Evaluation of Genotoxicity using Amphibian Larvae (International Organisation for Standardization) (ICS: 13.060.70, 2006; GENOVA-CH; http://iso.org/iso/ iso_catalogue/catalogue_tc/catalogue_detail.htm? csnumber $=39680$ )

[24] Mouchet F, Landois P, Sarreméjean E, Bernard G, Puech P, Pinelli E, Flahaut E and Gauthier L 2008 Characterisation and in vivo ecotoxicity evaluation of double-wall carbon nanotubes in larvae of the amphibian Xenopus laevis Aquat. Toxicol. 87 127-37

[25] Mouchet F, Landois P, Puech P, Pinelli E, Flahaut E and Gauthier L 2010 CNT ecotoxicity in amphibians: assessment of multi walled carbon nanotubes (MWNT) and comparison with double walled carbon nanotubes (DWNT). Special focus on environmental toxicity of nanoparticles Nanomedicine 5 963-74

[26] Bourdiol F, Mouchet F, Perrault A, Forquaux I, Datas L, Gancet C, Boutonnet J-C, Pinelli E, Gauthier L and Flahaut E 2013 Biocompatible polymer-assisted dispersion of multi walled carbon nanotubes in water, application to the investigation of their ecotoxicity using Xenopus laevis amphibian larvae Carbon 54 175-91

[27] Bour A, Mouchet F, Verneuil L, Evariste L, Silvestre J, Pinelli E and Gauthier L 2015 Toxicity of $\mathrm{CeO}_{2}$ nanoparticles at different trophic levels effects on diatoms, chironomids and amphibians Chemosphere 120 230-6

[28] Moldovan M S, Bulou H, Dappe Y J, Janowska I, Bégin D, Pham-Huu C and Ersen O 2012 On the evolution of Pt nanoparticles on few-layer graphene supports in the hightemperature range J. Phys. Chem. C 116 9274-82

[29] Pirzado A A, Jouane Y, Le Normand F, Akilimali R, Papaefthimiou V, Matei Ghimbeu C and Janowska I 2014 Electrical transport in 'few-layer graphene' film prepared by the hot-spray technique: the effect of thermal treatment J. Phys Chem. C 118 873-80

[30] Nieuwkoop P D and Faber J 1956 Normal tables of Xenopus laevis (Daudin) (Amsterdam: North-Holland)
[31] Madre J F 2006 Logiciel Mesurim. Académie d'Amiens (http://ac-amiens.fr/pedagogie/svt/info/logiciels/ Mesurim2/Index.htm)

[32] McGill R, Tuckey J and Larsen W 1978 Variations of box plots Am. Statist. 32 12-6

[33] Wynn T A, Chawla A and Pollard J W 2013 Macrophage biology in development, homeostasis and disease Nature 496 445-55

[34] Orecchioni M, Bedognetti D, Sgarrella F, Marincola F M, Bianco A and Delogu L G 2014 Impact of carbon nanotubes and graphene on immune cells J. Transl. Med. 12138

[35] Seabra A B, Paula A J, de Lima R, Alves O L and Durán N 2014 Nanotoxicity of graphene and graphene oxide Chem. Res. Toxicol. 27 159-68

[36] Zhang Y, Ali S F, Dervishi E, Xu Y, Li Z, Casciano D and Biris A S 2010 Cytotoxicity effects of graphene and single-wall carbon nanotubes in neural phaeochromocytoma-derived PC12 cells ACS Nano 43181-6

[37] Matesanz M C, Vila M, Feito M J, Linares J, Gonçalves G, Vallet-Regi M, Marques P A and Portolés M T 2013 The effects of graphene oxide nanosheets localized on $\mathrm{F}$-actin filaments on cell-cycle alterations Biomaterials 34 1562-9

[38] Yue H, Wei W, Yue Z, Wang B, Luo N, Gao Y, Ma D, Ma G and Su Z 2012 The role of the lateral dimension of graphene oxide in the regulation of cellular responses Biomaterials 33 4013-21

[39] Russier J, Treossi E, Scarsi A, Perrozzi F, Dumortier H, Ottaviano L, Meneghetti M, Palermo V and Bianco A 2013 Evidencing the mask effect of graphene oxide: a comparative study on primary human and murine phagocytic cells Nanoscale 5 11234-47

[40] Sanchez V C, Jachak A, Hurt R H and Kane A B 2012 Biological interactions of graphene-family nanomaterials: an interdisciplinary review Chem. Res. Toxicol. 25 15-34

[41] Li Y, Yuan H, von dem Bussche A, Creighton M, Hurt R H, Kane A B and Gao H 2013 Graphene microsheets enter cells through spontaneous membrane penetration at edge asperities and corner sites Proc. Natl Acad. Sci. USA 110 12295-300

[42] Seong S Y and Matzinger P 2004 Hydrophobicity: an ancient damage-associated molecular pattern that initiates innate immune responses Nat. Rev. Immunol. 4 469-78

[43] Zanni E, De Bellis G, Bracciale M P, Broggi A, Santarelli M L, Sarto M S, Palleschi C and Uccelletti D 2012 Graphite nanoplatelets and Caenorhabditis elegans: insights from an in vivo model Nano Lett. 12 2740-4

[44] Pretti C, Oliva M, Di Pietro R, Monni G, Cevasco G, Chiellini F, Pomelli C and Chiappe C 2014 Ecotoxicity of pristine graphene to marine organisms Ecotoxicol. Environ. Saf. $101138-45$

[45] Guo X, Dong S, Petersen E J, Gao S, Huang Q and Mao L 2013 Biological uptake and depuration of radio-labeled graphene by Daphnia magna Environ. Sci. Technol. 47 12524-31

[46] Gollavelli G and Ling Y C 2012 Multi-functional graphene as an in vitro and in vivo imaging probe Biomaterials 33 2532-45

[47] Mouchet F, Landois P, Datsyuk V, Puech P, Pinelli E, Flahaut $\mathrm{E}$ and Gauthier L 2011 International amphibian micronucleus standardized procedure (ISO 21427-1) for in vivo evaluation of double-walled carbon nanotubes toxicity and genotoxicity in water Environ. Toxicol. 26 136-45 